\title{
Fuzzy based Filtering Approach on Histogram Specification for Fingerprint Image Enhancement
}

\author{
Dr.S.Arumuga Perumal \\ Prof. \& Head, Dept. of \\ Comp.Science, S.T.Hindu \\ College, Nagercoil, TN, India- \\ 629002.
}

\author{
T.C. Rajakumar \\ Assistant Prof., Dept. of \\ Comp. Science, St.Xavier's \\ College, Tirunelveli, TN, \\ India-627007.
}

\author{
Dr.N.Krishnan \\ Prof \& Head, Dept.of IT, \\ M.S.University, Tirunelveli, \\ Tamilnadu, India-627012.
}

\begin{abstract}
Fingerprint identification is the most important area in biometric identification. The quality of the fingerprint image is an important one for a reliable matching process. The contrast of the image can be enhanced at the preprocessing stage of fingerprint matching. Contrast is the difference between two neighboring pixels. In this paper we describe a fuzzy modeling approach which may be used for reducing the noise and increaseing the brightness of the ridges. Fuzzy filter values are analyzed and better results are produced in the image domain. The probabilities of gray values are determined from the position of the input image pixel. The result indicates the good performance of the proposed fuzzy histogram.
\end{abstract}

Keywords : Image Enhancement, Contrast, Fuzzy Logic, Fuzzy Statistics, Fuzzy Filter, Histogram, Mean, Variant, Probability Density Function(PDF), Cumulative Density Function(CDF).

\section{INTRODUCTION}

Fingerprint identification is a prominent method for personal identification. Most of the verifications are based on minutiae matching. The image enhancement algorithm can improve the clarity of ridges and valleys. The singular point region is the region where the ridge position is higher than normal structure[13]. Gabor filter enhances the image on its orientation, but it is difficult to be accurate at the ridge position[7]. Filter estimation may be used to enhance the quality of the image. Linear filters are used for noise removal, edge detection, segmentation etc.[11]. Histogram processing is a non-linear contrast enhancement. The histogram of the original image is redistributed to produce a uniform population density of the image[3]. The different filter masks are used in the enhancement algorithm, but the resultant image may not be smooth and the unwanted pixels are also present. In the fingerprint image system, Gaussian noises occur during finger pressure. The proposed algorithm reduces the noises and the ridges are extracted in a smooth manner. In the following sections, we describe in detail our enhancement algorithm. Section 2 addresses the fingerprint enhancement techniques with filter mask. Fuzzy statistics on digital image and its process is given in section 3. Experiment result of fingerprint images with Histogram Equalization and its PSNR(Peak Signal to Noise Ratio) values are given in section 4 . Section 5 contains the summary of the fingerprint enhancement method.

\section{FINGERPRINT ENHANCEMENT TECHNIQUES}

The objective of fingerprint enhancement technique is to process an input image $\mathrm{I}(\mathrm{x}, \mathrm{y})$ and the result is more suitable for identification. The enhancement algorithm reduces the noises from the input image. The input image $I(x, y)$ is defined as a $M * N$ matrix, where I $(i, j)$ represent the gray of the pixel at the ith row and jth column. The first step of the fingerprint processing is normalization. Normalization is used to remove the effect of sensor noise and finger pressure difference. The normalization $\mathrm{N}(\mathrm{i}, \mathrm{j})$ for the image $\mathrm{I}(\mathrm{i}, \mathrm{j})$ with mean $\mathrm{M}$ and variant $\mathrm{V}$ is given below[12],

$$
N(i, j)=\left\{\begin{array}{l}
D+\sqrt{\frac{D R\left(I(i, j)-M^{2}\right.}{V}} \text { if } I(i, j)>M \\
D-\sqrt{\frac{D R\left(I(i, j)-M^{2}\right.}{V}} \text { otherwise }
\end{array}\right.
$$

$$
\text { where } \quad M=\frac{1}{N^{2}} \sum_{i=0}^{N-1} \sum_{j=0}^{M-1} I(i, j) \text {---------(2) }
$$

and

$$
V=\frac{1}{N^{2}} \sum_{i=0}^{N-1} \sum_{j=0}^{M-1}(I(i, j)-M(I))^{2}
$$

The parameters D and DR are the desired mean and variant values.

Filtering approach is a way of pursuing the pixel values from its surrounding locations.[1] The mean and the standard deviations of such neighborhood is used for contrast enhancement. Median filtering has been used for smooth image, with average weighted value depending on the neighboring pixels.

Frequency transformation decomposes an image from its spatial domain intensities into a frequency domain[2]. The frequency transformation shows the frequency of pixel brightness variations, pattern change and the amplitude of the signal waveform. Frequency domains are also performed for selective removal of noise pattern from an input image. 


\section{FUZZY STATISTICS ON FINGERPRINT IMAGE}

Fuzzy sets are capable of representing the statistical value based on the theory of Fuzzy sets [15]. A gray scale transformation may succeed in preserving edges in one image and it may fail in another one. Fuzzy statistical values can improve the quality of the input image by reducing noises and increasing the intensity slices. Fuzzy set A for digital images is defined in the ordered pairs

$$
A \equiv\{(x, \mu A(x)) \mid x \in U\}
$$

where $\mu \mathrm{A}(\mathrm{x})$ is called the membership function for the set of all values $\mathrm{x}$ in $\mathrm{U}[8]$. The membership values $\mu$ are permitted in the interval $0 \leq \mu \leq 1$, crisp set is consequently a special case of a Fuzzy set, with membership values restricted to $\mu \in\{0,1\}$. Fuzzy membership values are assigned from the following fuzzy function[4,5]

$$
\mu A(X)=\frac{\operatorname{Max}(0,1-|x-n|}{\alpha}
$$

Where $\mathrm{n}$ and $\alpha$ are the positive real values generated from the input histogram image.

\section{FUZZY BASED HISTOGRAM COMPUTATION}

In fuzzy set, the image can be written in the following form[14].

$$
A=\frac{\boldsymbol{U}_{i, j}}{f_{i, j}} m * n
$$

$$
\text { Where } \frac{\mu_{i, j}}{f_{i, j}} \quad 0 \leq \mu_{i j} \leq 1
$$

If $\mu_{i j}=1$, it indicates that the pixel is bright and when $\mu_{i j}=0$, then the pixel is dark.

The histogram of a digital image with gray levels in the range $[0, \mathrm{~L}-1]$ is a discrete function with $\mathrm{h}[\mathrm{rk}]=\mathrm{nk}$, where $\mathrm{rk}$ is the gray level value and $\mathrm{nk}$ is the number of pixels with gray level intensity $\mathrm{k}$ in the input image $\mathrm{I}(\mathrm{x}, \mathrm{y})[6]$. Histogram $\mathrm{h}[\mathrm{k}]$ is occurrence probability(frequency) of gray level $\mathrm{k}$ in an image.

$$
h(k)=\frac{n(k)}{n}
$$

where $\mathrm{n}$ is the total number of pixels. Transforming intensities so as to obtain a desired(specified) shape of histogram of output image is called histogram mapping[9,10]. $\quad P_{x}(U)$ is the continuous Probability Density Function(PDF) for the given image and $\mathrm{P}_{\mathrm{z}}(\mathrm{U})$ is the specified(derived) PDF for output image. The equalization of the given image $\mathrm{I}(\mathrm{x}, \mathrm{y})$ is

$$
Y=f(x)=\int_{0}^{x} p x(u) d u
$$

The histogram transformation of the specified image is given below,

$$
Y^{\prime}=g(x)=\int_{0}^{z} p z(u) d u
$$

\section{PROPOSED \\ METHOD \\ FOR FINGERPRINT IMAGE ENHANCEMENT}

The Fuzzy statistical values are determined on the basis of the gray intensity of the spatial domain. The image intensity is transformed with the specified Probability Density Function(PDF). The Cumulative Density Function(CDF) is used in accumulating pixel values in an ordered manner for stretching the contrast[3]. The smooth fingerprint image with less noise is produced in the output domain. The Fuzzy statistical value $\left(\mu_{x i}\right)$ of the input image and the inverse transform $(\mu y k)$ are used for histogram mapping, which is given in the hypothetical Fuzzy histogram specification diagram. The specification values[max $\left.\left(\mu_{x i}-\mu_{y k}\right)\right]$ are specified in the dark region, which is also shown in the figure (1). The Fuzzy statistics values $\left(0 \leq \mu_{i, j} \leq 1\right)$ and $\left(0 \leq \mu_{i, j} \leq 1\right)$ are used for constructing the smooth fingerprint image.

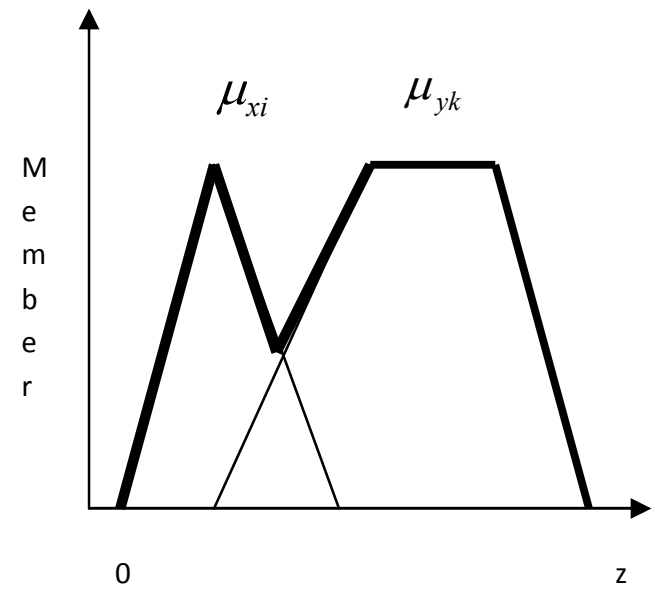

Fig. 1 A Hypothetical Fuzzy Histogram Specification

De-fuzzification process is performed with the fuzzy statistical value for achieving the enhanced specification image. The following stages are performed during the fuzzy histogram process for the fingerprint enhancement. 


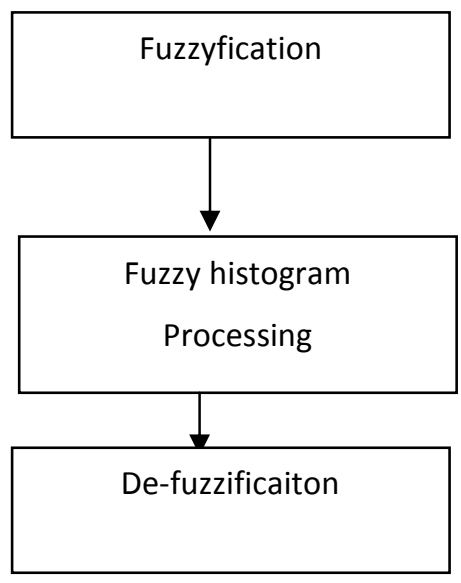

Fig. 2 General flow of Fuzzy Fingerprint enhancement

In the fuzzy maximization process, the discrete fuzzy gray intensity values are cumulated and the result is better than the classical Histogram Equalization(HE).

\section{EXPERIMENTAL RESULT}

In this section, we demonstrate the experiment for removing the blur background pattern from the input fingerprint image. The original arch fingerprint image is in figure 3 (a) and its histogram equalized image with PSNR $=38.1590$ is in figure 3(b). Figure 3(c) gives the proposed fuzzy histogram fingerprint image with clear background(valleys)). The PSNR value for the proposed method is 34.4963 . The fuzzy histogram graph is given in figure 3(d). Here the noises are suppressed and the ridges are clearly identified. Similarly in figure 4(a) the original whorl fingerprint image is given. The histogram equalization image with PSNR $=37.8282$ is given in figure 4(b). Figure 4(c) shows the fuzzy histogram fingerprint image with PSNR value 30.7969 and its graph is in figure 4(d).
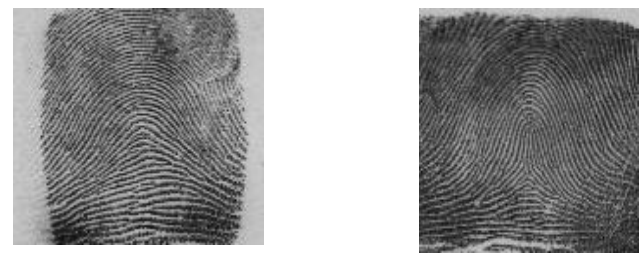

Fig. 3(a) Arch fingerprint

Fig. 4(a) Whorl fingerprint
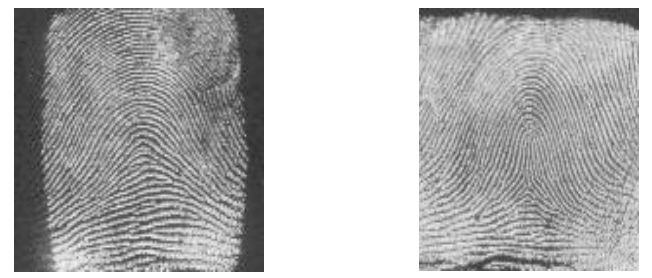

Fig. 3(b) Histogram Equalized Equalized image

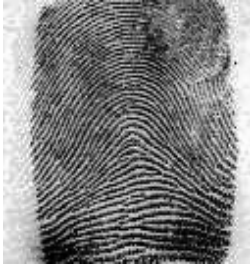

Fig. 3(c) Proposed Fuzzy Histogram image

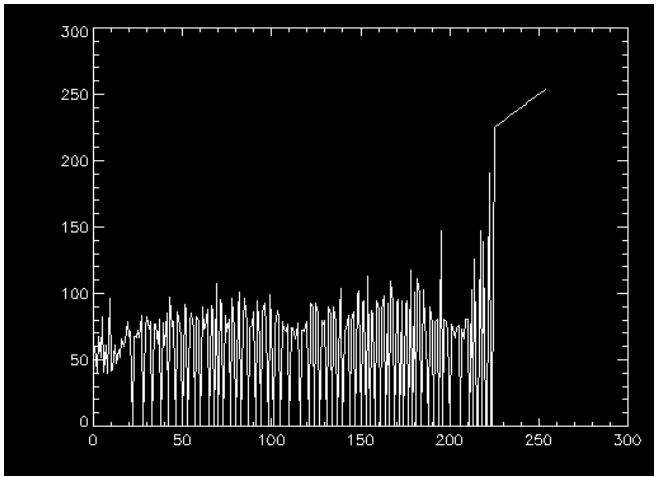

Fig. 3(d) Proposed Fuzzy Histogram

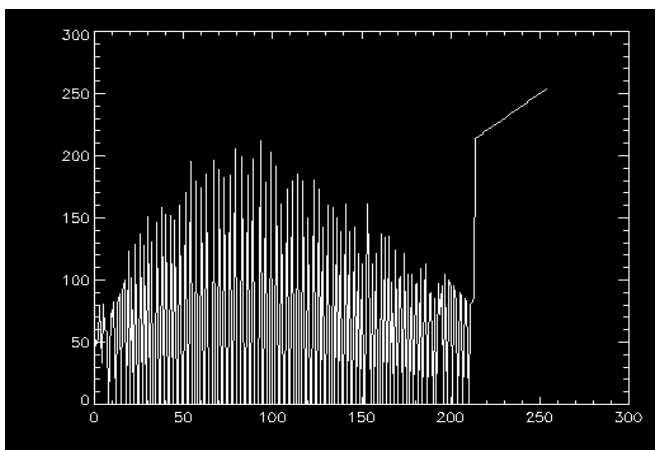

Fig. 4(d) Proposed Fuzzy Histogram graph Histogram graph

\section{CONCLUSION}

Our paper describes the concept of fuzzy histogram processing for contrast enhancement for fingerprint images. The proposed fingerprint enhancement algorithm is to improve the clarity of ridges and valleys of the input fingerprint image. Fuzzy logic provides a different approach on histogram specification for image enhancement. The fuzzy histogram generates intensity values, which may be used to improve the contrast of the input image. In the traditional filtering methods can not deal with the narrow intensity gray values. This method solves the problem of narrow and wide gray range images. The proposed algorithm is tested with several types of fingerprint images and the result shows that the generated images are improved in quality with less noise. 


\section{REFERENCES}

[1] Danielsson P E and Ye Q Z. 1988, "Rotation-Invariant Operators Applied to Enhancement of Fingerprints", Proc. Ninth ICPR, pp. 329-333, Rome.

[2] Daugman 1985 , "Uncertainty Relation for Resolution in Space, Spatial-Frequency, and Orientation Optimized by Two Dimensional Visual Cortical Filters", J. Optical Soc. Am., Vol. 2, pp. 1,160-1,169.

[3] Dinu Coltuc, Philippe Bolon and Jean Marc Chassery 2007, "Exact Histogram Specification", IEEE Trans., Image Processing, Vol. 15.

[4] Dong Liang Peng, Tie-Junwc 2002, “A Generalized Image Enhancement Algorithm Using Fuzzy Sets and its Application", IEEE Trans., Machine Learning and Cybermetrics, Beijing., pp. 820-823.

[5] Dubois D and Prade H. 1980, "Fuzzy Sets and Systems", Theory and Application, New York: Academic.

[6] Gonzalez R Wood. R 2009, Digital Image Processing, pearson Edn.

[7] Hong L, Wan Y and Jain A 1998, "Fingerprint Image Enhancement:Algorithm and performance Evaluation", IEEE Trans. Pattern Anal. Machine Intell., Vol. 20, pp 777789, Aug.
[8] Jawaher C.V and Ray A.K. 1996 "Fuzzy Statistics of Digital Images", IEEE Signal Processing Letters, Vol. 8., August.

[9] Kundu. S. 1998 "A Solution to Histogram Equalization and other related problems by shortest path methods", "Pattern recognit., Vol.31, no. 3, pp. 231-234.

[10] Pal S K 1980, King R A., Image Enhancement Using Fuzzy Sets, Electronics Letters, 16(10), pp. 376-378.

[11] Saint Marc P et. al. 1991, "Adaptive Smoothing: A General Tool for Early Vision", IEEE Transactions on Pattern Analysis and Machine Intelligence, Vol. 13, No. 6, pp 514529 , June.

[12] Sen Wang, Yangsheng Wang. 2004, "Fingerprint Enhancement in the Singular Point Area", IEEE Signal Processing Letters, Vol. 11, no. 1, January.

[13] Srinivasan V S and Murthy N N 1992, "Detection of singular points in fingerprint images", Pattern Recognit., Vol. 25, no.2,pp. 139-153.

[14] Yang. X and Toh P S. 1995, “Adaptive Fuzzy Multilevel Median Filters", IEEE Trans. Image Processing, Vol.4, PP 680-682.

[15] Zadeh L A. 1973, "Outline of a new approach to the analysis of Complex system and Decision Process", IEEE Trans. Syst., Man, Cybern., Vol. SMC-3, pp 28-44. 